\title{
Non-invasive Acoustic Sensing of Belowground Wooden Tissues: Possible Application to Spatial Mapping of Soil Usage by Tree Roots
}

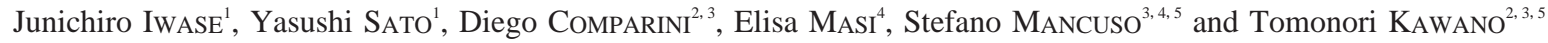 \\ ${ }^{\prime}$ Collaboration center, Kyushu Institute of Technology, Kitakyushu,Fukuoka 808-0196, Japan \\ ${ }^{2}$ Faculty and Graduate School of Environmental Engineering, The University of Kitakyushu, Kitakyushu, Fukuoka 808-0135, Japan \\ ${ }^{3}$ University of Florence LINV Kitakyushu Research Center (LINV@Kitakyushu), Kitakyushu, Fukuoka 808-0134, Japan \\ ${ }^{4}$ LINV-DiSPAA, Department of Agri-Food and Environmental Science, University of Florence, Sesto Fiorentino (FI), Italy \\ ${ }^{5}$ Univ Paris Diderot, Sorbonne Paris Cité, Paris Interdisciplinary Energy Research Institute (PIERI), Paris, France
}

(Received December 18, 2014; Accepted February 8, 2015)

\begin{abstract}
Growth of roots are central to the physiology and ecology of terrestrial higher plants including forest trees and agricultural crops. Ecologists, ecohydrologists, and biogeochemists need detailed insights into belowground properties and processes where plant roots take place, including changes in water, salts, and other elements that can influence ecosystem productivity and functioning. While growth and development of root system are dynamic, its status is hardly understood since roots grow belowground thus invisible with any optical means. In this study, we attempted to apply acoustic means for detection of undersoil tree roots, by developing a novel non-invasive sensing technology for detection of belowground plant tissues based on sound propagation in the soil. Novel instrumental set-up was designed for modulation and demodulation of sound signals passed through soil or sand with and without wood or tree roots. We detected the presence of wooden cut pieces and a living tree root both hidden belowground. This first attempt may induce further studies and developments by scientists and engineers for belowground sensing and imaging techniques as novel ecological research tools.
\end{abstract}

Keywords : sensing technology, sound modulation and demodulation, tree roots

\section{INTRODUCTION}

Ecologists, ecohydrologists, and biogeochemists need detailed insights into belowground properties and processes where plant roots take place, including changes in water, salts, and other elements that can influence ecosystem productivity and functioning (Jayawickreme et al., 2014). However, limitations in data and some confusions over terminology, together with a strong dependence on a small set of conceptual frameworks, have limited the exploration of root function in terrestrial models (Smithwick et al., 2014). Relying on traditional sampling and observation techniques for such insights can be costly, time consuming, and infeasible, especially if the spatial scales involved are large (Jayawickreme et al., 2014). Geophysical imaging provides an alternative or complement to traditional methods to gather subsurface variables across time and space, thus demands for sensing technologies enabling such belowground imaging are increasing to date (Jayawickreme et al., 2014).

Street trees are an integral element of urban life, which provide a vast range of benefits in residential and commercial precincts, also supporting healthy communities by providing environmental, economic and social benefits (Mullaney et al., 2015). In ecological point of view, root production and lifespan, root biomass, and rootmycorrhizal interactions govern the soil carbon fluxes and resource uptake, and are critical components of terrestrial models (Smithwick et al., 2014). However, increasing areas of impermeable surface in the urbanized environment can increase the stresses placed upon street trees and urban forests (Mullaney et al., 2015). Empirically we view that such stresses leads to imbalanced proliferation of tree roots towards the sites providing relatively favorable conditions for growth, often causing pavement uplift and damages to infrastructures nearby plants. Therefore, it is important to understand the spatial distribution of undersoil roots of the forest trees or street trees using non-invasive manner.

Here, we developed a novel non-invasive sensing technology for detecting of belowground tree roots based on sound propagation in the soil. We aimed at detection of tree roots hidden underground by using novel instrumental set-up which was specifically designed for modulation and demodulation of sound signals passed through soil or sand with and without wood or tree roots. Although the present attempt accomplished only the first simple test case, this may induce further studies and developments by scientists and engineers for belowground imaging techniques as ecological research tools.

\section{MATERIALS AND METHODS}

Acoustic sensing system

Modulation of sound signal through sand with and without wooden samples were monitored using a pair of

Corresponding author: Tomonori Kawano (plant biological issues), fax: +81-93-695-3304, e-mail : kawanotom@kitakyu-u.ac.jp 
acoustic probes, a flat sound radiator and sensing microphones (Fig. 1A, B). According to the specifications provided by manufacturer, propagation of sound by a flat sound radiator is unidirectional. In order to eliminate the background sound noise, signal from the reference (ground) microphone was used. For generating modulated sound signal at frequency of interest, a function generator (DFG602020 MHz DDS, EZ Digital Co., Ltd., Gwangju, Korea) was used (Fig. 1C). Signals received on sensing microphone was amplified with AT-MA2 Microphone amplifier (Audio-Technica, Tokyo, Japan) and band-filtered for demodulation using Multi-function filter 3611 NF Corporation (Yokohama, Japan), and the amplitude of the sound at specific frequency captured through the system was analyzed using an Oscilloscope (TBS 1064, Tektronix Inc., OR, USA).

Plant materials and plant supporting medium

Sandy soils are ideal for a wide range of plants. As a model plant supporting media, river sand purchased from local market was used. Bed of sand (volume, $4 \mathrm{~L}$; area 220 $\mathrm{mm} \times 262 \mathrm{~mm}$, depth, $100 \mathrm{~mm}$ ) filled with river sands with known water content $(0.98$ or $10.28 \%)$ was used.
Pieces of wood (columns, $\phi 23 \mathrm{~mm}$, or $\phi 50 \mathrm{~mm}$ ) derived from Asopalav Tree or Mast tree (Polyalthia longifolia Sonn.) known as Mempisang in Malaysia and Asoka in India, were used as model wooden pieces in the preliminary experiments for tuning the system for acoustic root sensing. A standing tree of Bay laurel (Laurus nobilis L.) was used as a model tree for detecting the belowground hidden roots. Density of wooden pieces was $c a .0 .62-0.75 \mathrm{~g} / \mathrm{cm}^{-3}$ that of sandy soil was between $1.39-1.47 \mathrm{~g} / \mathrm{cm}^{-3}$.

\section{RESULTS AND DISCUSSION}

Preliminary study using wooden pieces: Effects of sand humidity and size of object undermined

As preliminary laboratory scale experiments for selecting the range of sound frequency prior to monitoring of tree roots hidden belowground in the field, simplified experimental set-up inside the sand-filled container of polystylene foam (sand bed) with and/or without model wooden pieces (Asopalav tree, columns, $\phi 23 \mathrm{~mm}$, or $\phi 50$ $\mathrm{mm}$ ) inserted in the sand, was used as shown in Fig. 1. Then, band-modulated sound signals were emitted and detected at constant distance of $100 \mathrm{~mm}$, by a pair of acoustic
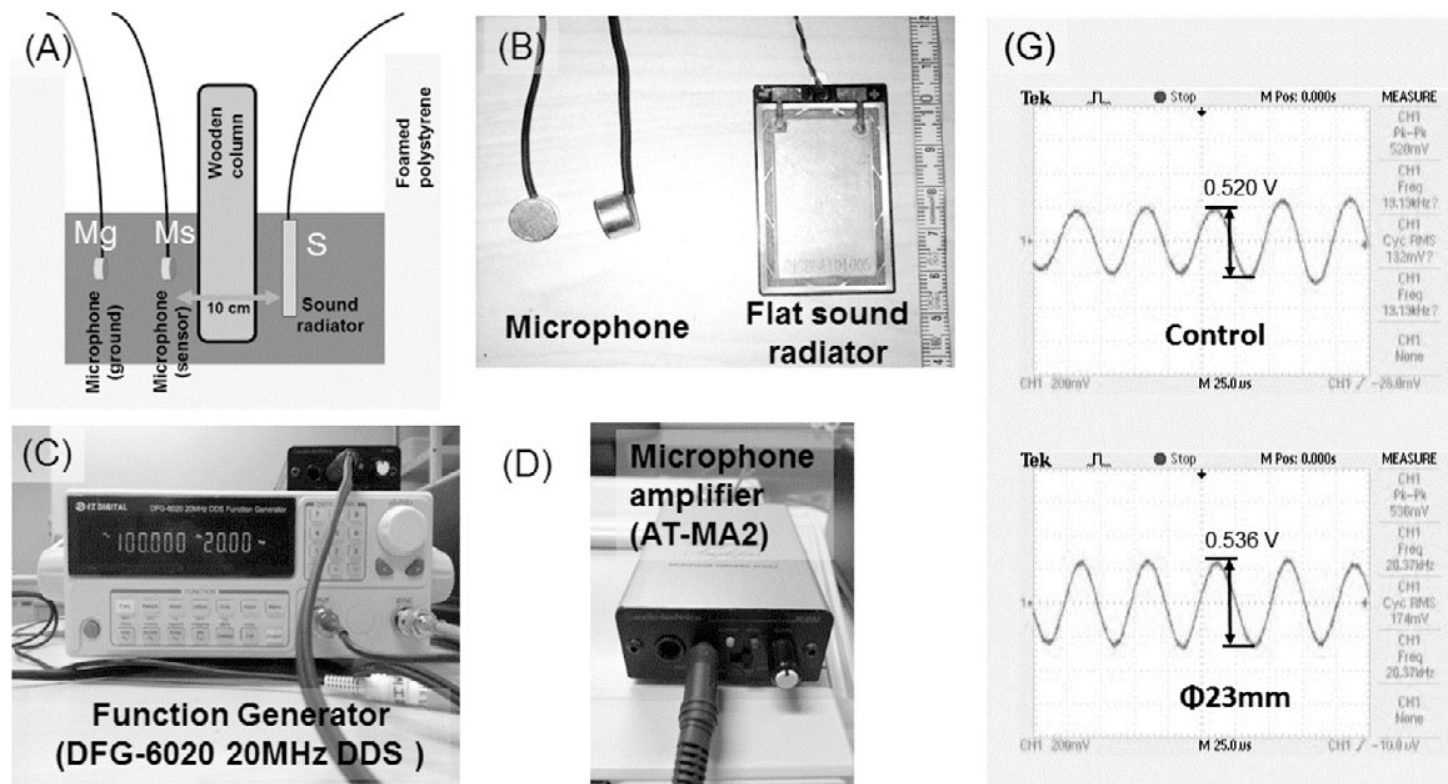

(D)
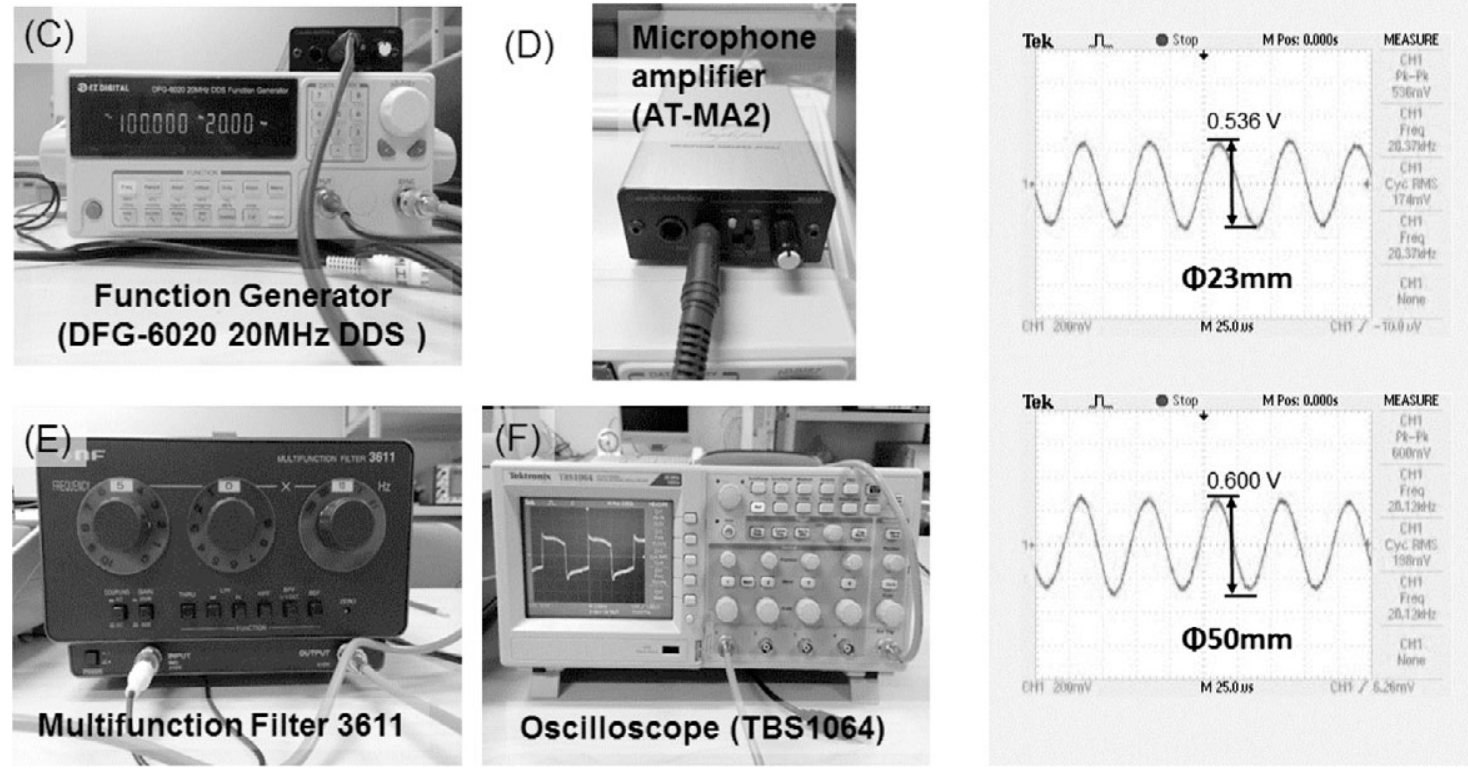

Fig. 1 Equipments consinsting the acoustic sensing of under-soil roots. (A) Experimental configulation. (B) A pair of acoustic probes designed for emitting and receiving the sound signal in the soil or sand. Left, sensing and reference microphones. Right, flat-shaped sound radiator with directional sound propagation. (C) Function generator used for generation of continuous sound signal with known frequencies between 100 and 20,000 Hz. (D) Microphone amplifier connected to microphones embedded in the soil or sand. (E) Multifunction filter used for band-filtering allowing elimination of noise. (F) Oscilloscope used for recording and analysis of sound signals. (G) Typical raw records of signal obtained at ca. $20 \mathrm{kHz}(19.19-20.37 \mathrm{kHz})$. Voltage changes between the peaks and valleys of the wave were expressed as signal intensity. 


\section{ACOUSTIC TREE ROOT DETECTION}

probes, namely, a directional sound radiator and a receiver (microphone), both enbeded in the bed of dry ( $\mathrm{ca} .1 \%$ of water content) or wet ( $c a .10 \%$ of water content) sands.

Acoustic signals modulated and demodulated between a pair of probes were assessed in two types of model sandy soil differed in water content (dry and wet, ca. $1 \%$ and $10 \%$ of water content, respectively). Intensity of sound signals propagated in the sand layer largely differed depending on the given sound frequency between 100 and $20,000 \mathrm{~Hz}$ (Fig. 2A, B). Compared to dry sand, wet sand likely enhanced the yield of signal of sounds (especially between 200 and 2,000 Hz).

To evaluate the wood-dependent modulation of sound signal, two indices were proposed. However, even through the use of indices, presence of wooden piece could not be detected by application of relatively lower range of frequency $\left(100^{-} 2,000 \mathrm{~Hz}\right)$ since signal intensity was not significantly altered by the presence of wooden pieces (data not shown). With the index of ratio (sample / blank) (Fig. $2 \mathrm{C}, \mathrm{F}$ ), increase in the yields of signal along with the increase in wood size (in diameter) were recorded at $4 \mathrm{kHz}$ (under wet condition) and $20 \mathrm{kHz}$ (both under wet and dry conditions). Similarly, with the index of subtraction (sample - blank) (Fig. 2 B, E), increase in the yields of signal along with the increase in wood size were recorded at 20 $\mathrm{kHz}$ (both under wet and dry conditions). Therefore, this range of frequency $(4-20 \mathrm{kHz})$ were chosen for demonstration of live root detection in the field.
Detection of living tree roots

In order to demonstrate the detection of roots hindered under the soil, we need to non-invasively perform detection of the presence of the roots, but for the test, manual evaluation must be done by removing the soil. Therefore, we obtained a permission to dig up around the roots of a standing tree (Bay laurel, Laurus nobilis L.) from the owner of the tree.

A living root of Bay laurel growing $40 \mathrm{~mm}$ beneath the soil surface was chosen for the study, thus a root was excavated and size was measured $(37 \mathrm{~mm}$ in diameter, Fig. $3 \mathrm{~A}, \mathrm{~B})$. The root was re-embedded in the sandy soil with known property (water content, 1\%). Level of covering sand was $40 \mathrm{~mm}$ above the top surface of the roots. By spanning the range of $40-45 \mathrm{~mm}$ below the sand surface, acoustic probes were inserted (Fig. 3C). Figure 3D and E show the configuration and experimental set-up for the measurement.

Detection of acoustic signals in the sandy soil with and without hidden living tree root was performed with acoustic probes. To assess whether the indices proposed in the laboratory scale test are applicable to evaluate the significant increase in signals or not, 10 measurements in the blank configuration and 10 measurements with the root were alternatively repeated for total of three sets (Fig. 4A). Mean value in each set ( $n=10 /$ set, totally 3 sets $)$ was used for evaluation of two indices (Fig. 4B, C). With both indices, measurements at $4 \mathrm{kHz}$ sound showed highest scores.
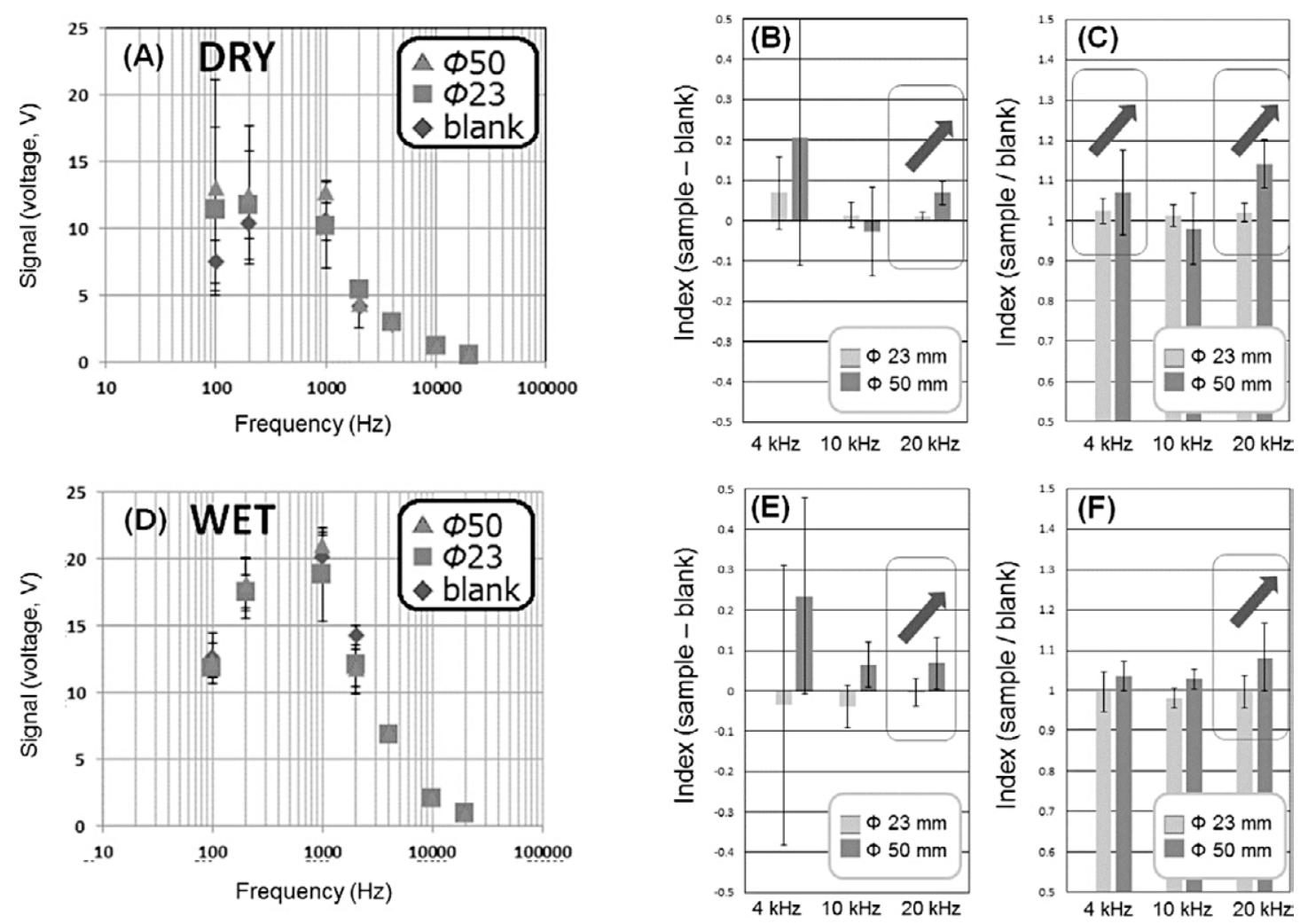

Fig. 2 Detection of sound signals propagated in the sand layer. Relatively dry (water content, ca. 1\%; A, B, C) and wet (water content, ca. $10 \%$; D, E, F) sands were used for evaluation. Relationships between the frequency and yield of sound signals in the beds of dry (A) and wet (D) sand are compared. Two indices, namely, index of subtraction (sample - blank) (see B, E) and index of ratio (sample / blank) (see C, F), were proposed for evaluation of wood size-resolution. Evaluations of indices were performed at higher range of frequency. Error bars, S.D. $(n=3)$. 

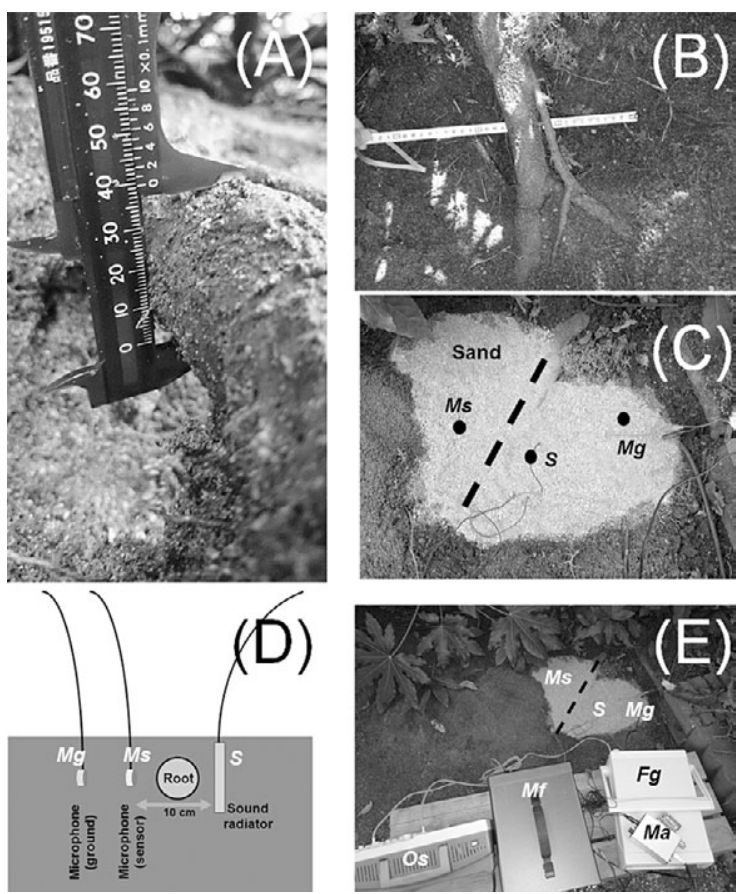

Fig. 3 Site of standing tree root detection. (A) A growing root of Bay laurel (sized $37 \mathrm{~mm}$ in diameter), excavated out at $40 \mathrm{~mm}$ below the soil surface was chosen for the study. (B) Site of excavation. (C) For model experiment, the soil on-site was replaced with the sandy soil of known property (water content, 1\%). Black dots indicate the positions of acoustic probes. (D) Configuration of experiment. (E) Experimental set-up on-site. Abbreviations used: $\boldsymbol{F g}$, Function generator; $\boldsymbol{M a}$, Microphone amplifier; $\boldsymbol{M f}$, Multifunction filter; $\boldsymbol{M g}$, ground microphone; $\boldsymbol{M} \boldsymbol{s}$, sensing microphone; $\boldsymbol{O} \boldsymbol{s}$, Oscilloscope; $S$, sound radiator. Broken lines indicate the position of the hidden tree root $(\mathrm{C}, \mathrm{E})$. For measurement of blank signal, positions of $\boldsymbol{M s}$ and $\boldsymbol{M g}$ were reversed and direction of sound propagation was also adjusted towards $\boldsymbol{M S}$ by repositioning (rotation) of $\boldsymbol{S}$.

$20 \mathrm{kHz}$ measurements also gave positive scores with both indices. In contrast, at $10 \mathrm{kHz}$, presence of root was hardly detected.

\section{Further studies}

Growth of roots are central to the physiology and ecology of terrestrial higher plants including forest trees and agricultural crops. The rhizosphere priming, an early step in root system development, is a mechanism by which plants interact with soil functions (Cheng et al., 2014) and soil microbes such as mycorrhizal fungi (Martin et al., 2001). While growth and development of root system are dynamic, its status is hardly understood since roots are growing belowground thus invisible with any optical means. In this study, we attempted to apply acoustic means for detection of undersoil tree roots for the first time. It is obvious that further studies and developments are needed especially to improve the accuracy and to enhance the spatial resolution. In addition, temporal resolution must be testified through continuous monitoring of growing roots. This technic may benefit the scientists and engineers in the area of root-centric eco-physiology, since the importance of root lifespan to whole-plant and ecosystem processes is
(A)

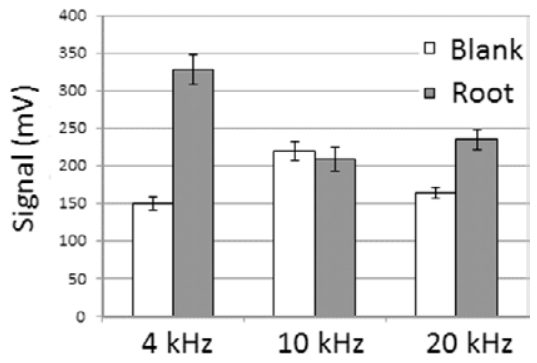

(B)

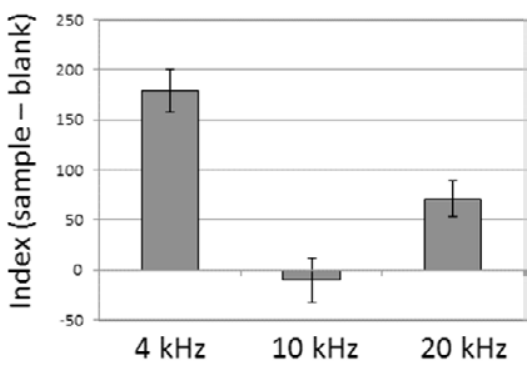

(C)

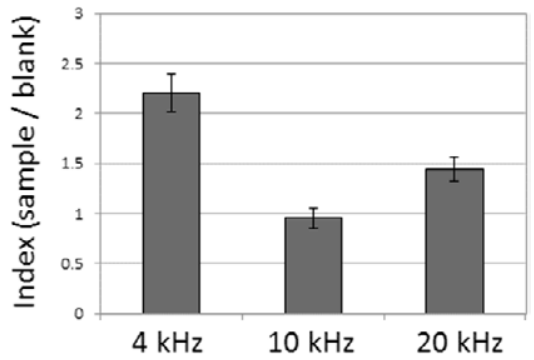

Fig. 4 Detection of belowground live tree root using acoustic probe. (A) Detection of acoustic signal in the soil with and without hidden living tree root. Blank signal was obtained with mock measurement without tree roots. (B) Evaluation of the signals with the subtraction index (sample-blank). (C) Evaluation of the signals with the rational index (sample / blank). Error bars, S.D. $(n=3)$.

increasingly recognized with concerns to the roots' dynamic process and response to changing climatic and edaphic factors (McCormack and Guo, 2014).

\section{Perspectives}

In the present study, we succeeded in detection of wooden pieces and a living tree root hidden belowground based on the changes in sound propagation in the soil with and without wooden/root samples. Based on the principles performed here, development of devices or equipment specifically designed for outdoor field measurements must be carried out. Since the yield of signal is highly sensitive to the conditions of soils such as water content as demonstrated in Fig. 2, we need to develop a database for casespecific calibrations according to the changes in water content and type of soil. In fact, we are now engaged in such innovative program with financial support as stated in Acknowledgement.

\section{ACKNOWLEDGEMENT}

This work was supported by Program for Creating STartups from Advanced Research and Technology (START Program) by The Ministry of Education, Culture, Sports, Science and Technology (MEXT), Japan. 


\section{ACOUSTIC TREE ROOT DETECTION}

\section{REFERENCES}

Cheng, W., Parton, W. J., Gonzalez-Meler, M. A., Phillips, R., Asao, S., Mcnickle, G. G., Brzostek, E., Jastrow, J. D. 2014. Synthesis and modeling perspectives of rhizosphere priming. New Phytol. 201: 31-44.

Jayawickreme, D. H., Jobbágy, E. G., Jackson, R. B. 2014. Geophysical subsurface imaging for ecological applications. New Phytol. 201: 1170-1175.

Martin, F., Duplessis, S., Ditengou, F., Lagrange, H., Voiblet, C.,
Lapeyrie, F. 2001. Developmental cross talking in the ectomycorrhizal symbiosis: signals and communication genes. New Phytol. 151: 145-154.

McCormack, M. L., Guo, D. 2014. Impacts of environmental factors on fine root lifespan. Front. Plant Sci. 5: art. no. 205. Mullaney, J., Lucke, T., Trueman, S. J. 2015. A review of benefits and challenges in growing street trees in paved urban environments. Landscape Urban Plan. 134: 157-166.

Smithwick, E. A. H., Lucash, M. S., McCormack, M. L., Sivandran, G. 2014. Improving the representation of roots in terrestrial models. Ecol. Model. 291: 193-204. 